\title{
Correcting Loose Connections in Distribution Networks from Two Perspectives: Loss Reduction and Power Outage
}

\author{
Mehdi Izadi ${ }^{1}$, Farzad Razavi ${ }^{*}$, Ardalan Najafi ${ }^{3}$ \\ 'Department of Electrical Engineering, Saveh Branch, Islamic Azad University, Saveh, Iran; \\ mehdiizadi_eeikiu@yahoo.com \\ 2Department of Electrical, Computer and Biomedical Engineering, Qazvin Branch, Islamic Azad University, \\ Qazvin, Iran; farzad.razavi@qiau.ac.ir \\ ${ }^{3}$ Faculty of Electrical and Computer Engineering, Shahid Beheshti University, Tehran, Iran; Ar.najafi@mail.sbu.ac.ir
}

\begin{abstract}
This paper is a report on an attempt to study fixing loose connections in an actual distribution network in Iran (Qazvin Power Distribution Company) from two angles: reducing loss and minimizing the costs emanating from power outage. The results show that fixing loose connections will only be cost-effective if account is taken of both loss reduction and outage costs. This finding can be used in making appropriate policies on correcting loose connections. This work was fully funded by the Qazvin Electric Distribution Company under contract number 420.
\end{abstract}

Keywords: Correcting Loose Connections, Distribution Network, Loss Reduction, Power Outage

\section{Introduction}

Preservation of energy is of much importance when environmental issues, expensive fossil fuels, and costs of developing power plants are taken into consideration. This has led national governments to invest significant amounts of money in reducing loss of electrical energy, with the most attention being paid to the high amount of loss at the distribution level. Clearly, loss of the energy generated for sale costs power utilities dear.

Loss is a function of various factors, a summary of which was provided in $^{2}$ :

- Ohmic loss in the conductors of primary and secondary network

- Ohmic loss in the windings of distribution

- Iron loss in the core of distribution transformers

- Ohmic loss in service cables between secondary feeders and customers

- Ohmic loss in leakage currents of shunt equipment, such as insulators and arrestors.
A wide range of methods of reducing loss have been attempted over the past few decades ${ }^{2}$ presented a list of such methods at the distribution level:

- Reconductoring in primary and secondary feeders

- Reconfiguring feeders

- Using high efficiency distribution transformers

- Reducing secondary network length by adding and optimally placing distribution transformers

- Using distributed generation

- Placing subtransmission substations near load centers

- Load balancing

- Improving load factor

- Improving voltage profile.

A common cause of loss in distribution networks is loose connections which can result in faults and power outages. Correcting loose connections will reduce line resistance and loss. A review of the methods tried for this purpose is given below.

*Author for correspondence 
The authors of ${ }^{7}$ and $^{6}$ studied the effect of fixing loose connections on loss reduction via thermography and then attempted to fix 2200 loose connections in Hormozgan power network and as a result reduced power loss by 10.5 MW and the operating costs by $\$ 39$ million.

The role of loose connections in overheating electrical joints was discussed by (Zhou \& Schoepf, 2011), who asserted that this problem often brings about electric fires, arc faults, and arc flash in electrical systems.

Similarly, ${ }^{5}$ focused on the issue of loose connections causing local temperature rise and the resulting deterioration of insulation material and disruption of electrical service. They proposed wireless temperature monitoring to detect abnormality in power plant equipment in order to prevent upcoming supply interruption.

Early detection of loose connections as weak points in a distribution system in Egypt was the subject of a study by ${ }^{1}$. The proposed detection methodology drew upon measurements of ultrasound emissions from such connections.

The papers reviewed above were primarily concerned with the technical aspects of the problem of loose connections and failed to consider the financial damage caused to power systems by this problem, the cost of fixing such connections, and financial benefit resulting from this correction.

The authors of ${ }^{4}$ prioritized five ways of reducing power loss in an actual feeder from the point of view of operating costs: adjusting load balance, placing and sizing capacitors, replacing dilapidated conductors and transformers, correcting loose connections. An important point that failed to be noticed was the effect of loose connections on power outage. Also, they considered the loss factor to be 0.52 . However, the loss factor the present research was considered 0.4047 by measuring both power loss at peak and the energy loss in the year at issue.

The present research was an attempt to study fixing loose connections in an actual $20-\mathrm{kV}$ distribution network in Iran (Sharif-Abad Network, as part of Qazvin Power Distribution Company) from two perspectives: reducing loss and minimizing the costs resulting from power outage. For this purpose, an Objective Function (OF) was formulated and then minimized using the Genetic Algorithm (GA). The following items were considered in the OF: the cost associated with fixing loose connections, the benefit gained from loss reduction, and the cost brought to the network by failure to correct loose connections which might result in outage. This latter item particularly makes the present work innovative.

\section{Model Formulation}

Loose connections in power systems give way to errors and loss in the network. The most frequently used methods in the past research to identify these connections were thermography $y^{5,6,8}$ and measurement of ultrasound emissions ${ }^{1}$.

\subsection{Correcting Loose Connections ${ }^{4}$}

Correction of loose connections in the network was as follows:

- Using computer software, the length of the lines linking buses together was calculated.

- The existence of a connection at each end of each line was presumed.

- A connection was added if the line linking up two buses was longer than $480 \mathrm{~m}$.

- For each connection a gene was considered.

- Genes are binary, with GA Flag $=0$ meaning that the loose connection is not in need of correction, and with GA Flag = 1 implying that correction is necessary.

- The assumed number of connections is only true about single-wire lines. For three-wire lines, this number should be multiplied by three.

- If a loose connection is corrected, line resistance declines by 0.001 ohms, in accordance with Qazvin Electrical Distribution Company.

- Finally, to measure the total cost of correcting loose connections, the operating costs pertinent to the correction of each connection was multiplied by the total number of connections $\mathrm{Eq}(1)$.

$$
C_{\text {connection-1 }}=n_{\text {connection-1 }} \times p_{\text {connection- } 1}
$$

where

- $C_{\text {connection-1 }}$ : the cost of fixing loose connections (\$)

- $n_{\text {connection- } 1}$ : the number of loose connections to be fixed (the number of GA Flags equal to 1)

- $p_{\text {connection- } 1}$ : the cost of correcting each loose connection (\$/unit)

\subsection{The Benefit Gained from Reducing Loss ${ }^{4}$}

The benefit gained from reducing power loss is calculated through Equation (2). 


$$
\begin{aligned}
B_{\text {loss-reduction }}= & \left(P_{\text {loss-after }}-P_{\text {loss-before }}\right) \\
& \times 8760 \times L S F \times p_{\text {energe }}
\end{aligned}
$$

where

- $B_{\text {loss-reduction }}$ : the benefit resulting from loss reduction $(\$)$

- $P_{\text {loss-after }}:$ loss after the application of the method $(\mathrm{kW})$

- $P_{\text {loss-before }}:$ loss before the application of the method $(\mathrm{kW})$

- $L S F$ : loss factor

- $P_{\text {energe }}$ : price of energy $(\$ / \mathrm{kWh})$

It should be noted that after the method is applied, since $P_{\text {loss-after }}$ is smaller than $P_{\text {loss-before }}, B_{\text {loss-reduction }}$ is a negative value.

Loss factor is obtained using Equation (3):

where

$$
L S F=\frac{E_{L}}{T \times P_{L}}
$$

- $P_{L}$ : Power loss at peak load $(\mathrm{kW})$

- $T$ : Period in hours (8760 $\mathrm{h}$ in this research)

\subsection{Objective Function ${ }^{4}$}

$\mathrm{OF}_{1}$ was defined as Equation (4):

$$
O F_{1}=C_{\text {connection }-1}+B_{\text {loss-reduction }}
$$

where

- $C_{\text {connection- } 1}$ : the cost of correcting loose connections (\$)

- $B_{\text {loss-reduction }}$ : the benefit resulting from loss reduction (\$)

It is necessary to note that when the objective function is minimized by the GA, $C_{\text {connection- } 1}$ which is a positive value is minimized, and $B_{\text {loss-reduction }}$ which is a negative value is maximized. The flowchart of the $\mathrm{OF}_{1}$ is displayed in Figure 1.

\section{New Method: Correcting Loose Connections Considering Power Outage as Well as Loss Reduction}

This work was an investigation into loose connections in relation to loss reduction and power outage. The fact is that a loose connection can be either corrected or left

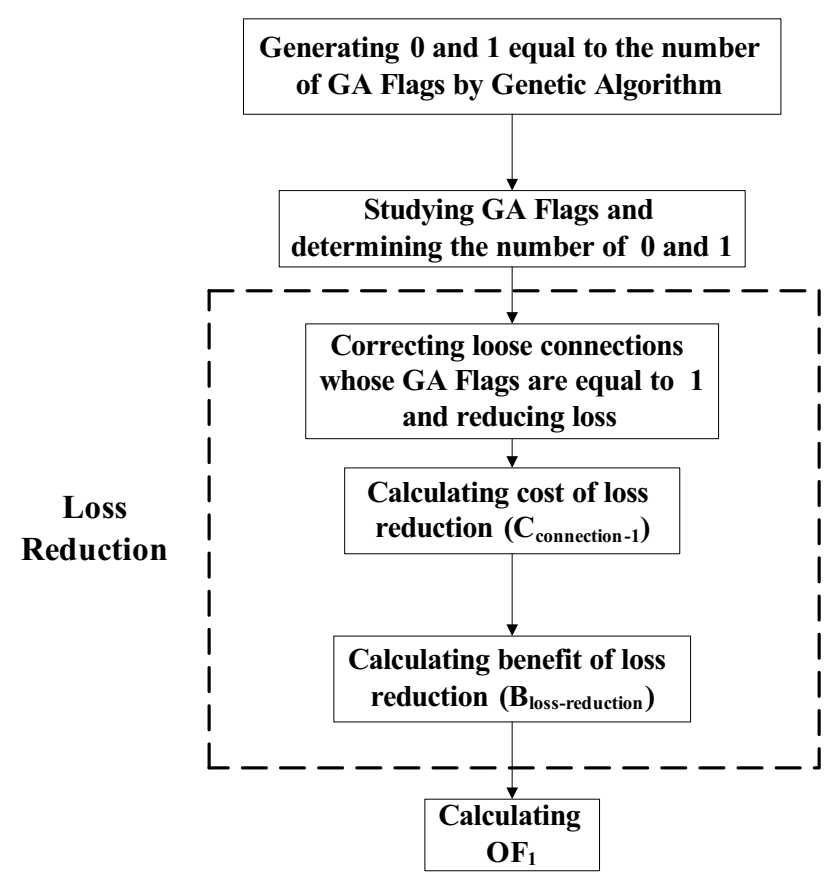

Figure 1. The flowchart of the $\mathrm{OF}_{1}$ for each iteration.

uncorrected. Correcting a loose connection will bring the benefit of loss reduction Equation (2), but it will also impose costs Equation (1). On the other hand, failing to fix a loose connection is potentially costly as it may lead to a fault in the network and thus to a power outage. In other words, if the electrical company leaves a loose connection uncorrected, it runs the risk of having to compensate its subscribers for the damage caused by the power outage. A model designed for calculating the cost associated with outage has to consider one or more of the following:

- the number of hours an outage lasts

- the amount of the load lost in percent

- whether the lost load was MV or LV

- the type of customer (i.e., agricultural, industrial, domestic, or commercial)

- the location where the load was lost relative to the feeder input

Determining the above-mentioned factors needs much research and is beyond the scope of the present paper. So, we assume that the cost imposed on the network by each loose connection is known. Accordingly, the cost of outage resulting from not correcting loose connections $\left(C_{\text {connection-2 }}\right)$ can be obtained from Equation (5):

$$
C_{\text {connection-2 }}=n_{\text {connection-2 }} \times p_{\text {connection-2 }}
$$


where:

- $n_{\text {connection-2 }}:$ the number of uncorrected loose connections (the number of GA Flags which are equal to 0 )

- $p_{\text {connection- }}:$ cost of outage resulting from leaving a loose connection uncorrected

Given that the purpose of the present research is to study correcting loose connections in terms of power outage as well as loss reduction, $\mathrm{OF}_{1}$ was reformulated as $\mathrm{OF}_{2}$ Equation (6).

$$
\mathrm{OF}_{2}=\mathrm{C}_{\text {connection-1 }}+\mathrm{C}_{\text {connection-2 }}+B_{\text {loss-reduction }}
$$

Figure 2 gives the flowchart of $\mathrm{OF}_{2}$.

The costs pertinent to correcting loose connections Equation (1) and possible power outages Equation (5) should be minimized, and the benefit to be gained from correcting loose connections Equation (2) should be

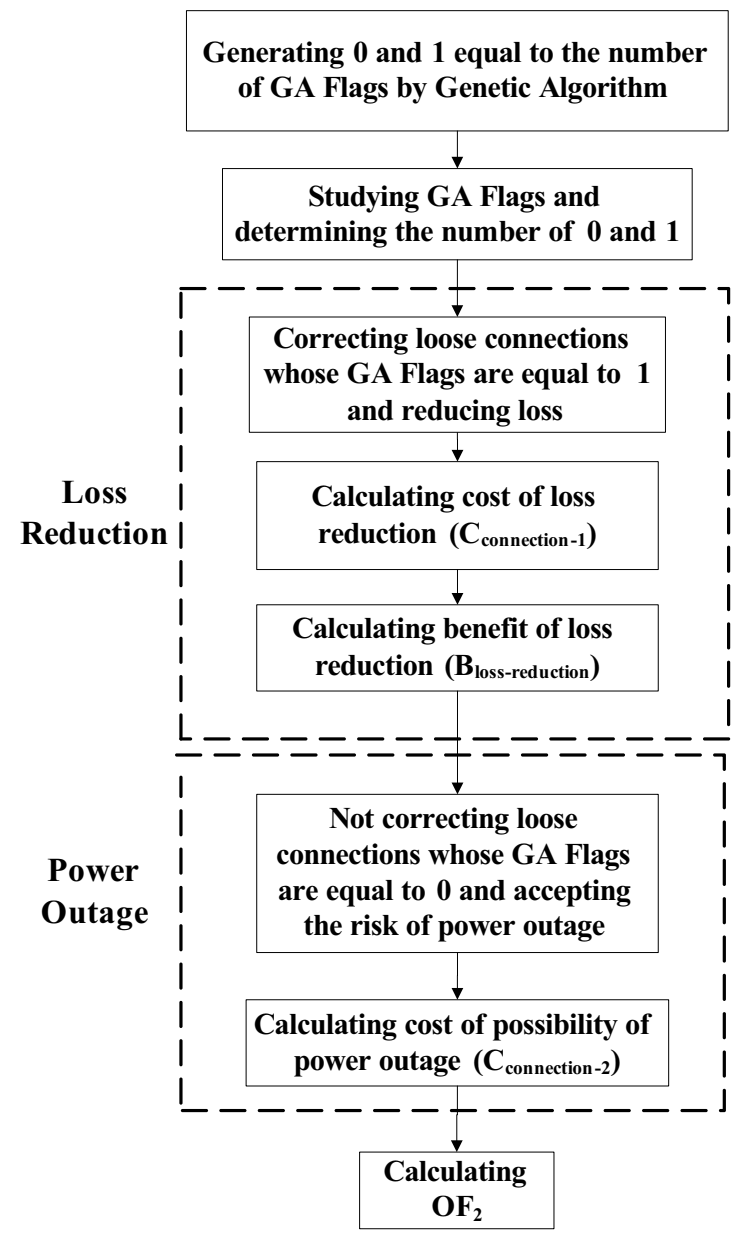

Figure 2. The flowchart of the $\mathrm{OF}_{2}$ for each iteration. maximized. This dual goal is achieved by minimizing $\mathrm{OF}_{2}$ The minimization tool used here was the GA. The result of putting the proposed method into use will be the best available option in this study because it shows the amount of costs associated with correcting or ignoring loose connections considering maximizing the benefit which can be gained.

\section{Simulation}

\subsection{Case Study}

The distribution network used in this study was the $20-\mathrm{kV}$ Feeder of Sharif-Abad in the northwest of Iran.

The features of this feeder can be seen in Table 1 and Table 2. It should be noted that it is necessary to know about the length of lines linking up terminals before we are able to calculate the number of loose connections in the system.

Table 1. A sample of the length of line between every two terminals in the network under study

\begin{tabular}{lc}
\hline Terminals i-j & Length $[\mathrm{km}]$ \\
\hline T59-T60 & 0.04658 \\
T60-T61 & 0.042101 \\
T62-T63 & 0.081154 \\
T64-T65 & 0.05293 \\
T65-T66 & 0.054265 \\
T66-T67 & 0.058357 \\
T67-T68 & 0.068757 \\
T68-T69 & 0.073169 \\
T69-T70 & 0.062034 \\
T70-T71 & 0.036182 \\
T71-T72 & 0.034367 \\
T72-T73 & 0.063003 \\
T73-T74 & 0.024233 \\
T74-T75 & 0.061842 \\
T75-T76 & 0.073207 \\
T76-T77 & 0.065371 \\
\hline
\end{tabular}

Table 2. The type of the conductors used

\begin{tabular}{lcc}
\hline $\mathrm{X}[\Omega / \mathrm{km}]$ & $\mathrm{R}[\Omega / \mathrm{km}]$ & Type \\
\hline 0.2464 & 0.2712 & 1 \\
0.2664 & 0.4545 & 2 \\
\hline
\end{tabular}


As reported by the Qazvin Electric Distribution Company, The price of a kilowatt of energy was $\$ 0.180$, and the cost of fixing a loose connection was $\$ 1.4058$ at the time of the study. As for the cost pertinent to leaving a loose connection unfixed, although we know for sure that failing to fix a loose connection would cost more money than correcting such a connection would, we cannot easily determine this cost (This problem was also discussed in Section 3 above). For this reason, considering the high reliability of the network under investigation and the compensation payable to customers in the event of an outage, we considered the cost of not correcting a loose connection to be ten times as large as the cost of fixing such a connection: $\$ 14.0580$. It should be reiterated that this amount (\$14.0580) is just a convention, meaning that the exact cost depends on the condition of the network at issue.

\subsection{Software}

DIgSILENT Power Factory 13.2 was utilized for the purpose of developing the proposed algorithm for the $\mathrm{OF}$ and analyzing the system. DIgSILENT is an advanced application for simultaneous analysis of power networks and control systems and thus is capable of measuring load flow, short-circuit level, active network losses, and parameters of the network. The most significant capability of this software is DPL (DIgSILENT Programming Language), which largely simplifies application of the proposed method. Optimization of the OF was made possible using GA on MATLAB R2008a Software. The two applications were connected to each other using a text file.

\subsection{Optimization}

For optimization, a population is defined. This population is formed via binary accidental quantification of chromosomes. The generated population is subsequently subjected to the $\mathrm{OF}$ in order to measure the fitness of chromosomes. Equation (7) presents the relationship between fitness and the OF.

$$
\text { Fitness }=\frac{1}{O F}
$$

Then, chromosomes should be selected from the present population for the reproduction purposes. Accordingly, two parent chromosomes are selected based on their fitness values, which are used later on by the genetic operators of crossover and mutation in order to produce two offspring for the new population. In crossover, genetic information between pairs, or larger groups, of individuals is exchanged. This work used two-point crossover for recombination. If crossover is the only operator used to produce offspring, we might face the problem that if every chromosome in the initial population has the same value at a particular position, every future offspring will also have this value at this position. To get over this problem, mutation is needed: a process which is aimed at randomly changing some of the genes. The current study used both operators to ensure optimization globalness ${ }^{3}$.

\subsection{The Proposed Algorithm}

In the proposed algorithm, GA determines what follows for each load:

- Each loose connection is given a value of either 0 or 1 , suggesting the necessity (1) or lack there of (0) of correction/replacement.

This is only done if constraints are not violated. The proposed method can be further specified below:

1) DIgSILENT writes 0 in the text file to flag the start of the initial calculation. Upon detecting this flag, GA will not start the relevant program.

2) DIgSILENT writes the matrix $\left[\begin{array}{c}1 \\ n_{\text {vars }} \\ \text { population_size } \\ \text { Generation }\end{array}\right]$ in the text file. The top row is the flag that shows the program should start to operate. When the flag is set to 1 , GA should run. $n_{\text {vars }}$ is the total number of the genes in the chromosome.

3) GA writes the matrix $\left[\begin{array}{llll}2 & X_{1} & \ldots & X_{k}\end{array}\right]$ in the text file. Here, $2 X_{1} \ldots X_{k}$ are the values of 0 or 1 related to loose connections of each line, and Flag 2 shows that DIgSILENT should restart its operation.

4) When DIgSILENT sees Flag 2 in the text file, it begins to operate and measures the OF using the chromosome in that file. Then, this software inserts Flag 3 and the quantity of the OF in the text file in the form of a matrix $\left[\begin{array}{c}3 \\ O F\end{array}\right]$, where Flag 3 denotes the temporary stoppage of DIgSILENT operation and the renewal of GA operation. 
5) If the maximum number of iterations is not reached, the above-said process turns to Stage 3. Otherwise, the process continues to Stage 6 described below.

6) The GA finishes and then inserts Flag 4 in the text file, marking the end of the process.

7) When DIgSILENT sees Flag 4 in the text file, it understands that the process is finished.

\section{Results and Discussion}

In this research, energy loss was found to be 505.295544 MWh, and active power loss turned out to be 0.142529 MW at peak. If we divide the amount of energy loss by that of active power loss, we would obtain the loss factor in this network for the year under investigation; 0.4047. Loose connections were corrected considering either loss reduction only or power outage as well as loss reduction.

\subsection{Running $\mathrm{OF}_{1}$ : Correcting Loose Connections Considering Loss Reduction Only}

Loose connections should not be corrected. The following explanation can be given:

As mentioned earlier on, the resistance of a loose connection in our study amounted to $0.0001 \mathrm{ohm}$. The resistance of the loose connections in a $0.480-\mathrm{km}$ line was $0.0003 \mathrm{ohm}$. The resistance of a $0.480-\mathrm{km}$ line was $0.11904 \mathrm{ohm}$. The resistance resulting from loose connections amounts to $0.08 \%$ of the total resistance of the line. The cost of correcting loose connections in a line will be $\$ 1.4058$.

There is a positive relationship between loss and resistance. The loss resulting from loose connections makes up $0.08 \%$ of the loss related to resistance. The loss emanating from network lines constitutes $45.18 \%$ of the total network loss. Hence, the loss caused by loose connections amounts to $0.036 \%$ of the total network loss. In other words, the total loss caused by loose connections is about $182 \mathrm{kWh}$, suggesting that the profit gained from reducing it will be almost $\$ 32.760$ per year. Considering the fact that the total number of loose connections in the network under study was 2514 , we will need $\$ 3534.1812$ to correct all those connections. The benefit which can be gained from correcting loose connections looks trivial in comparison with the costs involved.

\subsection{Running $\mathrm{OF}_{2}$ : Correcting Loose Connections Considering Power Outage as Well as Loss Reduction}

While running $\mathrm{OF}_{1}$ showed correcting loose connections not to be cost-effective, a glance through the data presented in Table 3 indicates that fixing will be cost-effective if account is taken of the power outage resulting from leaving loose connections unfixed. Loss reduction after running $\mathrm{OF}_{2}$ was $2.3408 \mathrm{~kW}$ at peak. In addition, the cost of correcting loose connections amounted to $\$ 410.4936$, and the cost of outage resulting from leaving loose connections uncorrected equated to $\$ 463.914$. The former turned out to be $47 \%$ of the total costs while the latter constituted $53 \%$ of the total costs. Clearly, the cost of correction is almost equal to that of leaving the connections uncorrected. However, the fact is that loss was reduced thanks to correction, with $\$ 1493.7402$ saved as a result.

Regarding the proposed method and given that failing to fix a loose connection is surely costlier than correcting such a connection, it can be said that fixing a loose connection is cost-effective only if both loss reduction and power outage are factored in.

\section{Conclusion}

This research studied the importance of loss reduction and power outage in correcting loose connections in an actual distribution network. A model was proposed for calculating the costs associated with each factor. The results show that correcting loose connections will only be cost-effective if both loss reduction and power outage are taken into consideration. This finding can help power

Table 3. Correcting loose connections considering power outage and loss reduction

\begin{tabular}{lc}
\hline Item & Quantity \\
\hline Loss after run [kW] & 142.816 \\
Loss reduction [kW] & 2.3408 \\
Cost of correcting loose connections [\$] & 410.4936 \\
Cost of leaving loose connections & 463.914 \\
uncorrected [\$] & \\
Total cost [\$] & 874.4076 \\
Benefit [\$] & 1493.7402 \\
$\left|\mathrm{OF}_{2}\right|[\$]$ & 619.3326 \\
\hline
\end{tabular}


utilities make the best policy when attempting to correct loose connections. A limitation of the proposed model is that outage costs were considered fixed. This means that future studies can develop a similar model with the outage costs being a function of the length of outage, percentage of the lost load, and type of customer.

\section{Acknowledgement}

This work was totally funded by the Qazvin Electric Distribution Company. Qazvin, Taleghani street, Qazvin Electric Distribution Company; Phone: +00982812239801-5; email: info@qazvin-ed.ir

\section{References}

1. Abdel-Salam M, Abdel-Sattar S, Sayed Y, Ghally M. Early detection of weak points in MEEC distribution system. Industry Applications Conference, 2001. Thirty-Sixth IAS Annual Meeting. Conference Record of the 2001 IEEE; 2001 Sep 30-Oct 4; Chicago, IL, USA.

2. Haghifam, MR, Malik OP. Genetic algorithm-based approach for fixed and switchable capacitors placement in distribution systems with uncertainty and time varying loads. IET Generation, Transmission \& Distribution. 2007; 1(2):244-52.
3. Hosseini SA, Karami M, Madahi SSK, Razavi F, Ghadimi AA. Finding the optimal capacity and location of distributed generation resources and analyzing the impact of different coefficient factors. J Basic Appl Sci Res. 2011; 1(12):2578-89.

4. Izadi M, Razavi F, Gandomkar M, Najafi A, Soleimani M. Power Loss Reduction in Distribution Systems through an Intelligent Method Considering Operational Costs. J Basic Appl Sci Res. 2012; 7(2):6744-56.

5. Livshitz A, Chudnovsky BH. Condition diagnostics of energized electrical equipment: case studies. Power Engineering Society General Meeting, IEEE; 2006; Montreal, Que.

6. Nemati G, Nasr KS. Asset Management: Condition based Monitoring Effect on Utility Coasts and Lose Power Consumption. 16th Conference on Electrical Power Distribution Networks (EPDC); 2011; Bandar Abbas.

7. Nemati, Gholamreza, Nasr, Salimi K. Reducing Operation Costs and Losses Using Thermography. 21st International Conference on Electricity Distribution; 2011; Frankfurt Germany. Availablefrom:www.cired2011.org/documents/ List-of-posters-S3-CIRED2011.pdf

8. Zhou, Xin, Schoepf T. Characteristics of Overheated Electrical Joints Due to Loose Connection. IEEE 57th Holm Conference on Electrical Contacts (Holm); 2011; Minneapolis, $\mathrm{MN}$. 